\title{
Human Digital Twins in Acquiring Information About Human Mental Processes for Cognitive Mimetics
}

\author{
Pertti SAARILUOMA ${ }^{\mathrm{a}, 1}$, Antero KARVONEN ${ }^{\mathrm{a}}$ and Lotta SORSAMÄKI ${ }^{\mathrm{b}}$ \\ ${ }^{a}$ University of Jyväskylä, Cognitive Science, Faculty of Information Technology \\ ${ }^{\mathrm{b}}$ Technical Research Centre of Finland Ltd. (VTT)
}

\begin{abstract}
Modern information technology makes it possible to redesign the ways people work. In the future, machines can carry out intelligence-requiring tasks, which previously were done by people. It is thus good to develop methodologies for designing intelligent systems. An example of such methods is cognitive mimetics, i.e. imitating human information processing. Today, machines cannot by themselves navigate in archipelagos. However, the fact that people can take care of ship steering and navigation means that there is an information process, which makes it possible to navigate ships. This information process takes place inside the minds of navigating people. If we are able to explicate the information processing in the navigator's mind, the knowledge of it can be used in designing intelligent machines. Replicating physical objects and industrial processes by means of digital computers is called digital twinning. Digital twins (DTs), which are digital replicas of physical systems and processes, have recently become tools for working with complex industrial processes. A crucial question for DTs is should human actions be added to them? As the answer is positive, such models of human information processing can be called human digital twins (HDTs). The knowledge of human tacit and explicit information processes can be represented by human digital twins. Models can be used in the search for a deeper understanding of human intelligent information processes. Human digital twins can thus be used as methodological tools in cognitive mimetics. In our present study, we modeled paper machine operators' thinking. Specifically, we developed an ideal-exception-correction (IEC) model for paper operators' control logic. The model illustrates how research and HDT-modeling can be used for explicating the subconscious or tacit information processing of people for the design of intelligent systems. In this article a model for design processes using cognitive modelling will be suggested. The concepts of cognitive mimetics and human digital twins enable us to outline a model for using the long tradition of simulating human thinking as a tool in designing intelligent systems.
\end{abstract}

Keywords. Digital Twins, Human Digital Twins, Cognitive Mimetics, Cognitive Modelling

\section{Introduction}

The emergence of intelligent technologies is becoming a sign of our time. Modern information technology makes it possible to redesign the ways of human work and carry out tasks people that used to do. Typical examples of innovative solutions can be found - for example, in transportation, in healthcare, as well as in industrial, legal, and

\footnotetext{
${ }^{1}$ Pertti Saariluoma; E-mail: ps@jyu.fi.
} 
administrative information processing [1][2][3]. People can be freed from many routine tasks, if only it is possible to innovate, design, and develop solutions taking care of human functional roles. The emergence of intelligent societies and artificial intelligence (AI) technologies is a central challenge of our times. Therefore, it is essential to develop methodological practices to enable designers to create working artefacts taking care of human roles in practical tasks.

Machines have always been designed, developed, and manufactured to enable people to reach their action goals [4][5]. People have their needs, which motivate their actions. In the beginning, there were only simple tools, such as hand axes or spears. However, by means of elementary technologies, it was possible to construct huge buildings, from palaces to pyramids [4]. After the development of precision work and steam energy, it was possible to begin industrialization, which used mechanical machines and later electromechanical systems. Finally, electromechanical technologies led to information technology with the rise of computing as the latest game-changer [6] [7] [8].

Industrialism changed the way people worked and lived. There is no doubt that information technology has already changed and will again change how people satisfy their needs and carry out work processes. One essential property of computational thinking is the possibility to create intelligent technologies, which can carry out intelligence-demanding tasks [9][10][11]. Traditionally, people have had to operate machines as there are numerous control decisions to be made, which could not be done by machines. Automatization, AI, autonomous technologies, and machine intelligence will free people from many present jobs, as they already have done. However, before it is possible to replace people in intelligence-demanding tasks with machines, it will be necessary to innovate, design, develop, and manufacture technological solutions capable of taking care of these tasks.

It is important to think about the foundations of how to design intelligent technological solutions. Here, we will begin with a simple idea. If people today are able to carry out some intelligence-demanding tasks, knowledge of this could be used to solve the problems of designing intelligent technologies for various contexts. If people can navigate ships through complex archipelagos, it is clear that there exists an information process that can carry out such a task. Logically, designers should be able to use the knowledge of the present information process to develop intelligent machines to take care of the same task.

In cognitive research, the idea that the same information process can be carried out by different physical entities is called "multiple realizability" [12]. For example, people are able to do mental calculation; however, pocket calculators can also effectively realize the processes of calculation. The focus or research should not thus be on the physical entities realizing information processes, but the information processes themselves. Information processes provide an independent level of conceptualization. The problem transforms into how designers can best acquire and use the knowledge of human information processes in creating intelligent technologies.

Our approach fleshes out a concrete question: how designers can use cognitive modelling of human thinking in design processes. Suggestions to this direction has been made sometimes in the past [13][14][15][16][17][18][19]. In this article a model for design processes using cognitive modelling will be suggested. The concepts of cognitive mimetics and human digital twins enable us to outline a model for using the long tradition of simulating human thinking as a tool in designing intelligent systems. 


\section{Content-based thinking}

It is possible to realize information processes in different physical systems from humans and animals to computers. The phenomenon has been termed "multiple realizability" [12]. The use of multiple realizability in designing intelligent systems is possible, if one knows how people process information in some particular tasks. A necessary presupposition for constructing a pocket calculator is to know how people process arithmetic information. The goal of our work is to understand how paper machine operators process information in their work. Our focus is on the contents of processed information and for this reason our approach has been called content-based analysis of human information processing [20][21] [5].

The roots of content-based thinking can be found in the history of cognitive science. Content-based thinking begins with Turing's [7][11] modeling of the mathematical mind and his idea that machines can process information like human beings. Newell and Simon [16] developed Turing's thinking. They assumed that people are information processing systems and unlike Turing [11], they began to study empirically how the human mind operates [16]. They modeled human information processes computationally and initiated a wide research on the role of capacity in human information processing [22], overviewed in the collection by [23].

However, the early tradition gave much more weight to limited capacity than to mental contents, because the analysis of limited capacity as an explanatory ground was highly successful in working with problems of human technology interaction [22] [24][25][21]. Furthermore, the figure of Shannon [26] and his information theory influenced this as well [27][8]. Shannon's information theory was essentially capacityoriented, as he deemed the contents of messages irrelevant (to the engineering problem) [26].

Newell and Simon [16], as well as many other researchers, saw the problem of mental content but paid much less attention to it than the problems of the limited capacity of human information processing. The focusing on mental capacity instead of mental contents has not been good for developing intelligent technologies, because the essence of human information processing is in its capacity to analyze, process and create new mental contents [21].

Some steps towards the analysis of mental contents can be found in concepts of cognitive simulation models, such as production systems [28][22][16], theories of mental models [29][30], and semantic networks [28][31]. From the present point of view, the use of these concepts has rather turned on the human limited capacity to have these entities in working memory and mind than on their information contents. On the other hand, the ground concepts created over the past four decades may in the future be effectively used in analyzing how people process mental contents.

Content-based analysis of information processing requires that the researchers are able to learn to understand the actual content of people's minds. This fact entails some prerequisites of the research process. Content-based analysis is different from those approaches that analyze human action on the external level. Content-based thinking focuses on the internal properties of information processes [16][20]. We do not deny the importance of the action level studies. These studies provide one element of understanding. Our purpose is, however, to point out the importance of internal information processes, which make it possible for people to guide and control, in this case study, paper machines. Ultimately, the explanation and the reason for particular actions is in the mental contents (conscious or unconscious) of actual human operators. 
Content-based analysis of human information processing has several phases. Firstly, it is important to collect raw data on information in the minds of operators in different situations. This can be done with a number of qualitative methods used to explicate mental contents [32][33]. Typical examples of data collection methods are protocol analysis [32], observation, discourse analysis, narrative methods, documentary analysis interviews, focus groups, and even qualitative tests [33]. The main goal of data collection is to get as good an idea as possible about how operators, or people in general, mentally represent their work situation and respective actions.

Explicating mental contents, i.e. information contents of mental representations is the core activity of content-based analysis [21][34]. The raw data must be turned into explicit descriptions of mental contents as this is the way one can use mental contents as explanatory ground for human actions. For example, if paper machine operators represent in their mind that the process runs too fast, they reduce the machine speed following their mental models for the situation [35]. If they misinterpret and misrepresent the same situation, it is possible that their actions will lead to operational failures. Thus, the mental contents of the operators make it understandable why they choose a nonoptimal manner of action. To be able to analyze and to explain such situations, researchers have to have a clear idea of the mental contents of operators in the particular situations.

An understanding of the action-relevant mental contents enables a researcher to study the digitalization of intelligent information processes. As people are able to carry out the intelligence-demanding task, knowledge of how people process information is vital for developing technical artefacts, which can carry out the same tasks.

\section{Cognitive mimetics in designing intelligent technologies}

Existing intelligent information processes can be used to develop new information processes in new physical entities. The process of developing new information processes on the ground of old ones can be called mimicking. Mimetic design, more broadly, is one important method of design today.

Mimicking nature is a well-founded branch in technology design [36]. Mostly its focus has been on creating physical entities by taking inspiration from the solutions found in nature. This kind of design thinking is called biomimetics or biomimicry. The main idea of biomimetics is to solve complex technical problems by imitating the solutions that nature has developed for similar problems. Many physical artefacts from clothes, spades, and airplanes to Velcro tape, have their origins in biomimetic design. However, in developing intelligent technologies, a new model of mimicking is required. Instead of mimicking biological structures, it is good to focus on human information processes and imitate them in developing intelligent systems. Mimetics based on an analysis of human information processes in developing intelligent technological solutions can be called "cognitive mimetics." [37]

The basic shape of any mimetic design process has a source $\mathrm{S}$, a target $\mathrm{T}$, and a mapping $(\mathrm{m})$ relation between them. Broadly speaking, for cognitive mimetics the source is the human mind and the target is a computer system. 


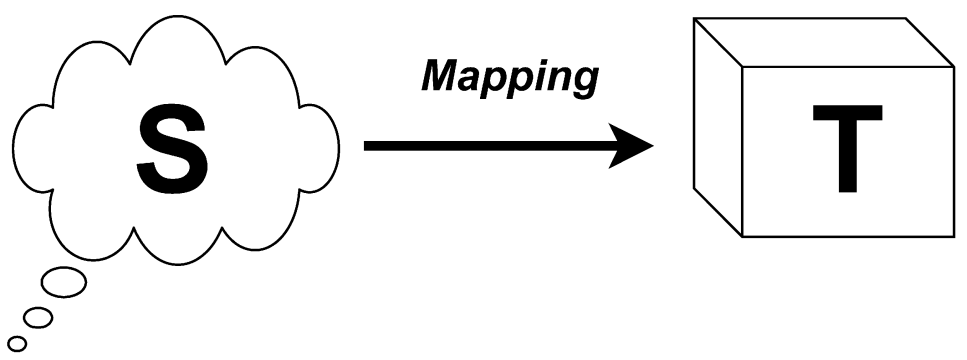

Figure 1. Mimetic mapping. S: Human mind, T: Simulation model.

Both the source and the target include here aspects such as context, environment, task, and task requirements. What the designer is looking for in mimetic design is, first, what makes the source (s) an effective solution in its context, and second, how to map (m) or transform that into a technological form $(\mathrm{t})$. Thus, the process can be understood as "an abstraction to concretization loop," where ideas are abstracted from the source and transformed into designs and concrete results.

An important aspect are the appropriate abstraction(s) of the source. A bird achieves flight (source) in the air (source environment) exploiting aerodynamic laws (abstraction of the coupling), for example. It achieves propulsion, lift and can control itself (abstract task requirements). From a mimetic perspective, these are among the right abstract ingredients for human flight, and yet, da Vinci's idea of copying the wing-flapping of birds was not the best path. This is the basic issue around multiple realizability in mimetic design - one does not get very far by direct copying, because the context of the target constrains possibilities in its own way (and indeed is constructed around those constraints). Importantly for us, the same is true for computers and intelligence. Computers have their own way of working and in the way they process information and mimetic solutions must be built around those constraints. Of course, nothing stops an inventive person from redesigning computers' operational principles through mimetics, but that is not our focus here. The crucial point for now is that the noetic resources, to borrow a term from [38], used by the human in completing intelligence-requiring tasks in a context, constitute the source for cognitive mimetics. The core issue that the mimetic design perspective brings to the surface is how the triplet source, mapping and target can be effectively achieved. This is at its' core the fundamental question for which we are seeking to model a particular solution for in this paper.

\section{Human digital twins in explicating tacit knowledge}

A difficulty with analyzing human information processing is that most mental contents are subconscious or tacit [39]. People are not aware of what they should do. Therefore, it is necessary to investigate the tacit levels of information in human mind in order to get a reliable and valid picture of how people process information during intelligencerequiring tasks. 
One way to obtain a deeper understanding is to get a clear method of explicating mental contents, and a good method for presenting how information processing works in the minds of people. In the research on human information processing and thinking, it has been thought that computer simulations might provide a means to represent what happens in human minds when, for example, they solve difficult problems [22][16].

Originally, the idea of imitating human thinking by means of computer programs was presented by Turing [7][11]. Actually, the basic model of computational algorithms has been the Turing Machine, which is a model of how mathematicians think [40][7]. After Turing, Newell and Simon [16], among many other researchers, began to develop the idea that people are some kind of computers [41].

Recent work on mimicking physical objects and industrial processes by means of digital computers has been called digital twinning. Digital twins (DTs), which are digital replicas of technical systems and processes, have recently become a tool for working with complex industrial processes [42]. They model components and functionalities of the systems and, therefore, support design, operational planning, and maintenance. A crucial question for DTs is how should human actions be analyzed? When are they relevant? How should human digital twins be modeled? From which level of abstraction should the human be analyzed? These questions and possibilities are a natural follow-up from the original DT prospect and have been identified in parallel by at least [43] [44] and [45]. As mimetic design shows, there are legions of different levels of abstraction' (LoAs) [46] one can take on the human. What is a rational and pragmatic level of abstraction is dictated by the purpose of the human digital twin (HDT). If the HDT is about physical ergonomics of a workplace, one is likely to look at anatomical models [42]. If, on the other hand, it is about human action with respect to technology, a good LoA could be the cognitive perspective. If the model is used to analyze fuel combustion processes in a turbine, user knowledge is of less relevance than in the case of thinking how the users control artefacts. As digital twins typically take a wide perspective into a technical system, it becomes likely that human action should often be included in the twins. For this reason, it is vital to discuss different approaches to modeling human actions as parts of digital twins.

Human digital twins (HDTs) are models of human actions when interacting with technologies. They can focus on narrow issues such as usability and even user experience, but there are no obstacles to modeling technical artefacts as parts of human work and human life. The issues of designing human machine collaboration processes can benefit from digital twinning, but they can also be used, for example, to explicate expertise and other types of tacit knowledge. This in turn can be used for artificial intelligence (as in cognitive mimetics) and many other purposes, like learning and developing organizational knowledge. In traditional engineering, such as building cyber physical systems, interacting with human actions can be seen as functional machine elements. This means that a number of machine functions are supposed to wait for input from users. Thus, car drivers have a set of controls they can use to get their car to behave as they want. In such examples, people are seen as a kind of input machine. However, it is also possible to study how it is possible for people to use technical systems. Usability, UX (user experience), and life-based design are typical examples of looking at humantechnology interaction through the concepts of cognitive and human research [5].

Human digital twins are likely to present the next frontier of digital twinning. We have here only scratched the surface of their possibilities. We will next outline through a case study one approach for human digital twinning, oriented around the mimetic perspective. One way to understand our approach is to see it as a first layer of intelligence 
on a digital twin of a factory process. It is about control [47] which is directly based on the empirically acquired mental contents of operators in a specific context. The model may appear simple, but its' function is to provide a demonstration how cognitive simulation can be integrated with design thinking as a way of explicating tacit knowledge.

\section{Our domain - paper machine operators' information processes}

Paper machines produce paper fast and in large quantities. They transform pulp suspensions, containing even more than $99 \%$ of water, to paper webs in seconds. The web is dried to $1.5-10$-meter-wide paper sheets with less than $.02 \mathrm{~mm}$ tolerance at a speed of over $80-90 \mathrm{~km} / \mathrm{h}$. Obviously, paper machines require high-precision engineering, and they are no less complex than big airplanes. Papermaking has a number of human-driven process parts, which may eventually be replaced by intelligent technology solutions. Interestingly, paper machines have a kind of mimetic origin: "The Fourdrinier machine represented a straightforward mechanization of what was formerly done by hand." [48]. Automation, more broadly, is the continuation of this same trend but it begins to encompass the further mimetics of the information processes, rather than manual labor of paper-machine operators. The first proportional-integral-derivative (PID) controller, for example, was a technical solution directly based on the anticipatory mental processes of a steersman in ships [49][50]. Our work here can be placed into this continuum by applying cognitive perspectives to the problems of creating digital intelligence for paper industry processes.

The studied environment was a pilot scale paper machine designed for research purposes. This means that in contrast to industrial-sized paper machines, paper grade changes and optimization of running parameters take place far more frequently (even several times within an hour). Interviews with the operators showed that this means that the process is run more manually than normal paper machines and therefore it requires more human thought and action. On the other hand, pilot runs are, in general, not so different from the operation of normal paper machines. In both cases, there are targets for the paper specifications and the operator's task is to align the process and the raw materials with those goals. However, in the pilot environment there may be fewer variables to consider and adjust than in normal paper machines. Here, the focus is typically on optimal conditions against a few - and even, in some cases, only one variables and there is flexibility with the rest. In industrial paper machines, the product must be as good as possible, and conditions of the run are secondary and may be suboptimal.

In the studied pilot paper machine environment, the operator works in a control room that is separated from the paper machine by a windowed wall. The process is operated and controlled through four medium-sized computer screens. The operator obtains information from the process by four principal means:

1. Graphical and numerical information from the Valmet DNA Distributed Control System (DCS) and the Trimble Wedge data analytics system. The Valmet DNA is a user interface that gives the operator the control of all processes. Through the DNA, the operator sees the prevailing process conditions (flows, levels of tanks, consistencies, pressures, etc.) and the operation status of the main process equipment (opening degree of valves, running of pumps, etc.), and is able to adjust/control the process. The Wedge 
data system, on the other hand, contains over 600 online measurement points from the pilot paper machine. With Wedge, the operator may follow the changes in the most important process parameters online as well as check the historical trends of the parameters.

2. Visual information from several cameras placed around the paper machine through a screen. The operator may also observe some parts of the process directly through the windowed wall.

3. Audio information (radio communication) from operators (2-3) in the field. The communication concerns mainly the tasks and task-status communication, and information transmission.

4. Audio information from the paper machine system. This is a largely tacit dimension where sounds, especially expected or anomalous, are used as an additional source of information. For example, the sound of a pump changes when the level of the tank it is pumping is below the level detectable by the level controller. This can be used as information to stop the pumping.

In the studied pilot paper machine, four operators run the process. Three of them operate in the field, i.e. in the immediate vicinity of the machine. Their tasks include manual procedures like sampling, opening valves, feeding the tank with fiber, etc. They communicate with the fourth operator via radio phones. The fourth operator operates in the control room and has the main responsibility. The operator controls and adjusts the process with the DNA system. He performs a set of actions during the start-ups and shutdowns of the machine, which is fairly routine. However, adjusting the process during the actual trial point to achieve the set targets for the end product or running parameters is not so straightforward. Achieving the specifications for the end product or a sufficient runnability of the machine with a totally new raw material requires thorough knowledge of how the machine works, the capability to exploit know-how and theory from earlier pilot runs, and also fast problem-solving skills. Even though every pilot trial point is unique, the operator utilizes the experience from earlier trials.

The research material was collected by video recording. The camera was positioned "over the shoulder" of the operator and mainly captured the screens that showed the process control system(s). The main idea was to capture sound, namely the think-aloud protocols of the operators. The operators $(n=2)$ were reminded to say what was on their minds and what they were thinking or watching. All in all, we captured approx. 7 hours of material, which was then transcribed into text and analyzed into episodes and phases. This analysis is still ongoing.

\subsection{Examples from the protocols}

We collected verbal think-aloud protocols on operators during work [32]. The main goal was to collect information about the basic structure of operators' thoughts in order to model them. The following presents an excerpt from protocol materials to demonstrate the highest-level structure of operators' actions.

\section{Example 1. The Fluctuating Flow of the Headbox Feed Pump Episode:}

$\mathrm{K} 1:[0: 20: 12]$ What are you looking at?

M1: [0:20:14] The speed of the middle ply alters quite a bit, sometimes it is 25 and then 34 . [pause $5 \mathrm{~s}$ ]

M3: [from the radio] [0:20:26] What do you mean by speed? 
M1: [responds] [0:20:31] I mean volume flow [pause $5 \mathrm{~s}$ ]

M3: [from the radio] [0:20:41] (inaudible) a lot of fluctuation (inaudible) by eyesight. [pause $11 \mathrm{~s}$ ]

M1: [responds] [0:20:54] 26-37, fluctuates between there.

M3: [from the radio] [0:21:01] Is air removal on? [pause $7 \mathrm{~s}$ ]

M1: [responds] [0:21:10] No, I'm putting it on. [thinks aloud] [0:21:12] So the air removal had been left off [pause $5 \mathrm{~s}$ ] [talks to radio] [0:21:23] it's on. [pause $12 \mathrm{~s}$ ]

M3: [from the radio] [0:21:38] (inaudible)

M1: [responds] [0:21:48] Yeah, let's see where it settles. [pause $10 \mathrm{~s}$ ] [talks to radio] [0:22:02] I'm going to decrease the flow of the headbox feed pump [pause $14 \mathrm{~s}$ ]

K1: [0:22:21] What are you thinking now?

M1: [thinks aloud] [0:22:26] That [I will decrease] the headbox feed pump, so that the flow doesn't go too high. [0:22:37] Now it is pretty good, fluctuates between 32 and 34, I will try to adjust the (inaudible) [pause $10 \mathrm{~s}]$ [0:22:55] That's why it was fluctuating, the air removal was not on. [pause 12s]

In a nutshell, the ideal state was violated by the fluctuating flow of the headbox feed pump. This resulted in a quick problem-solving episode, where the field operator suggested that it might be because the air removal was not on. Turning it on corrected the fluctuation.

The follow-up interview showed that this cause was "a classical one," accounting for $95 \%$ of cases in their estimation. The second "go-to" reason would have been blockage in the headbox hoses. The operators could list about 10 reasons for the event off the top of their heads.

\section{Example 2:}

M1: [thinks aloud] [0:43:33] Now the level in tank A is a bit high. I'm putting it on manual and reducing the level control, and then putting it back on automatic. [pause $9 \mathrm{~s}$ ] [0:43:55] Also I will decrease the chemical dosing (so that the density won't go too low.) [0:44:05] Also, I will manually put the dilution water valve to zero (-) [silent talking] to automatic. [0:44:19] Tank A shouldn't overflow, you can follow that from the camera. [0:44:22] If it overflows, you lose fiber. [0:44:29] Now the level is OK, density also pretty close [pause $7 \mathrm{~s}$ ] [0:44:42] It's coming down pretty fast [the density], I'm going to turn off the chemical pump so it doesn't go too low. [pause $8 \mathrm{~s}$ ] [0:44:58] Checking the density in tank B, the level in tank A is good, it is in the set point. [0:45:21] Flows are good, looks like the density in tank $\mathrm{A}$ is altering. [pause $11 \mathrm{~s}]$ [0:45:40] (--) I'm looking to see if I still need to do something about it. [0:45:47] No need, its going fine, it is 520 and (--) going down (-) and the (chemical) pump is off, so we are getting close to the trial point. [pause $11 \mathrm{~s}$ ] [0:46:09] The density in tank A has set to 520. [0:46:16] I have to monitor if it still starts to change (-) [pause $5 \mathrm{~s}$ ] [0:46:25] Still going down. [0:46:28] Then I must remember to decrease the flow of the headbox feed pump to 32 , it is still too high [pause $8 \mathrm{~s}$ ] [0:46:46] Density is good. [0:46:50] I'm going to decrease the flow of the headbox feed pump a little, just so that I reach the targeted flow. [pause $7 \mathrm{~s}$ ]

[talks to radio] [0:47:04] Densities are pretty much there. I'll still decrease the flow of the headbox feed pump, so we get the flow right, to 32 .

In both examples we can see the same structure. Operators recognize that something is not as it ideally should be. Therefore, they look for methods to correct the state of affairs. Interestingly, the contents of their thinking are not precisely in the ongoing situations, but they foresee how things will go wrong if they don't make their correcting operations. Thus, they anticipate possible future events and actions based on past and present information. This kind of anticipation has long been known to be typical for human thinking [51].

The operators' thought of processes on a higher level apparently take the form of ideal-exception- correction. They see how things are straying from the path they should be on and consequently they understand that they have to do something to prevent things 
from reaching that state. They compare their information on the present state of the process with the idea to find out means to reach an ideal state after correcting operations.

Based on the protocols we can see the basic logic of the operators' 'thinking. They have an ideal state in their minds. What the ideal state is depends on a number of issues, such as the quality of paper they are now producing, the raw materials they have at their disposal, and the state of the production process. Operators encode the present state of the technical process and register exceptions from the ideal. They can register deviations from the ideal state by comparing the present state with the expected ideal. Here it is important that operators can predict possible critical situations based on the indicators they have about the process in its present state and their own knowledge of the paper machine behavior. Finally, operators have in their minds a list of possible corrective actions, which they apply to bring the process to the ideal state.

\section{Modeling mind IEC_0.81}

In order to get concrete clarity on the use of a digital twin in acquisition of knowledge on mental contents, we developed a small model for operator information processing called IEC_0.81. The model is a very simple process model, but it contributes as it can be used to develop the use of human digital twins in acquiring information about human information processes in controlling paper machines.

IEC_081 assumes, based on collected empirical knowledge, that paper machine operators' thinking has an IDEAL-EXCEPTION-CORRECTION (IEC) loop. This means that operators observe the behavior of the machine process by means of measurement instruments and visual contact in the control room. Information is in addition passed "from the field" by other operators who work near the paper machine. When they observe an unexpected state of the process (or rather a deviation from the ideal), they make respective corrective actions following their models for corrective actions.

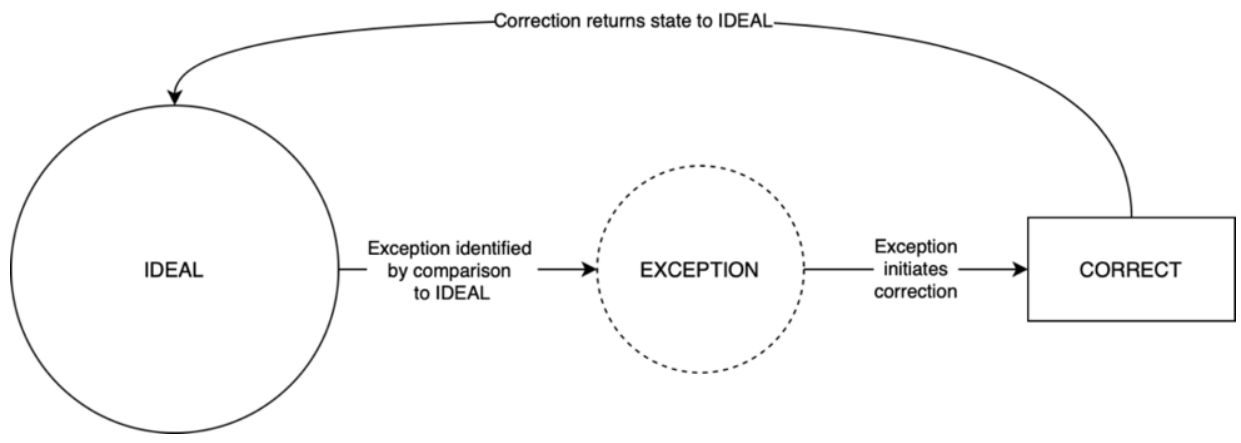

Figure 2: Ideal-exception-correction model.

The model is based on the idea that all human involvement points (HIPs) can be defined. Such a point is a place in a process in which people become involved in the process. The point entails a set of observation values (OVs) and a set of possible actions (PAs). Since the machines are closed and defined systems, they have for each HIP a limited set of OVs and PAs. All the possible human actions of involvement in the ongoing machine process can be thus defined in terms of HIPs, OVs, and PAs. 
In the case of analogue controls, which are in principle continuous, one can digitalize the operations. For example, a rudder that has an infinite number of positions can be divided into a finite number of possible states by means of assuming it is digitalized. As another example, in recordings the voice varies continuously, but it can be represented with sufficient accuracy in digital recordings. Thus, the basic analysis of a finite number of possible HIPs can be kept.

As noted, IEC 081 is very simple, but it can still give us an idea about the role of digital twin models in information collection. The model gives an interpretation for one possible solution to the problem of how human information processes and their content operate in controlling paper machines. The problem is that in its present elementary state, IEC_081 does not present the possible HIPs with sufficient accuracy. However, it can point out the open points in the control process and thus direct further collection of information. One can go to the operators and collect knowledge required in the description of HIPs. This in turn is what we have called the "mental contents."

IEC_081 does not yet have a detailed description of ideal processes, and ideal states, or corrective actions. It does not yet have detailed descriptions of detailed operator actions. Nevertheless, the model can be developed further by studying how operators carry out their actions in different situations. Thus, the model can very effectively aid in directing information collection on operators' mental contents.

The model also enables researchers to test the logic of their interpretation of data. If simulations work, this suggests that the interpretations do not have a problem in their formal structures. If simulations do not work, it means that the interpretations must be reanalyzed. Internally contradictory models cannot be possible and thus simulation makes it possible to perform a self-corrective analysis and interpretation of data on mental contents.

\section{Discussion: Computational thinking in mimetics}

Intelligent technologies are constructed conceptually on Turing machines [7]. The Turing machine is different from biomimetical artefacts as they are based on analogy between human and machine information processes. Turing machines do not imitate biological structures, but human thinking. Turing's machine was originally a machine version of how mathematicians process information [40][7]. As Turing machines need not limit themselves to mathematical information - for example, the symbols in them could be Chinese letters - they can also be used as models of the mind as an information processing system. Often intelligent technologies, whether theoretical or practical, have their origins in imitating how people process information. Cognitive information processing models describe how people process information [52].

To be able to imitate human information processes, designers need to know what these processes are like. Since vast parts of human information processes are subconscious or tacit, it is not easy to gain a solid understanding of what happens in the minds of people when they carry out some intelligence-requiring task. It is necessary to explicate the tacit information in order to acquire a sufficient understanding of how people are able to carry out these processes.

One method of explication is to construct digital twins, which are computational models of source processes. Here, the source processes would be human information processes and thinking. In basic research, much work has been done to computationally 
model the human information processing system [16][22]. Thus, simulative cognitive psychology can provide many tools for explicating human information processing and thus give ideas for developing intelligent information systems.

Interestingly, we found that modeling can guide empirical research. IEC_0.81 is a very primitive model for operators' information processing. It basically analyzes how operators detect exceptions and how they revise the processes into the ideal course of affairs. In the present conceptual version of IEC, it tests constantly the state of the machine process in HIPs or human interaction points. They are states in which operators have controls to regulate process. These points are places in which people become involved in the paper process and change them in some way. The ways they can do something is defined by machines.

In the model, all corrective actions are under one function called CORRECT. In order to move forward it is essential to study the mental contents that enable people to do various types of operations typical for CORRECT. Thus, the design of CORRECT functions enables modelers to focus their data collection on definable points in operators' information processing. This means that modeling proceeds through steps, in which researchers collect information about operators' mental contents. For example, the present version of IEC_ 0.81 has an operator called "control," but it does not yet analyze the methods of controlling and the connections between states of paper machine and respective controls. This analysis and modeling can be done in later versions. They reevaluate what has been modeled so far to be able to go further.

In data collection, researchers can use the same methodological thinking as above. Only the topic can specifically be focused on the relevant points in operators' information processing. The collection of information can again be based on qualitative methodologies typical to modern psychology [32][33]. The goal of the research process is to explicate tacit and explicit information contents in the minds of operating people.

The process outcome of designing intelligent information processes has an iterative structure. Empirical research of human information processes and especially their information contents are used to model what happens in human minds during some specific information process. The model can be used to direct information collection, and mimetically in generating technical systems that can carry out the tasks.

The main outcome of our research is to show how simulation of human thinking can be used as a part of the designer's thinking and coping with tacit knowledge. Thus, the IEC model is a demonstration of how explicate tacit human information processes for human designers working with intelligence-requiring information processes in industrial systems. Our goal has been to outline a practical approach for designing intelligent information processes from a cognitive mimetics approach [37]. Cognitive simulation models have their role in explicating tacit and subconscious processes within existing human work systems and operationalizing this knowledge in intelligent system design.

\section{References}

[1] Ford M. Rise of the Robots. New York: Basic Books; 2015.

[2] Fukuda K. Science, technology and innovation ecosystem transformation towards society 5.0. International Journal of Production Economics. 2020; 220: 3-14.

[3] Tegmark M. Life 3.0. Harmondsworth: Penguin Books; 2017.

[4] Bernal JD. Science in History I-VI. Harmondsworth: Penguin; 1969.

[5] Saariluoma P, Cañas J, Leikas J. Designing for Life. London: Macmillan; 2016. 
[6] Minsky ML. Computation: Finite and Infinite Machines. Englewood Cliffs, NJ: Prentice-Hall; 1967.

[7] Turing AM. On computable numbers, with an application to the entscheidungs problem. Proceedings of the London Mathematical Society. 1936-1937; 42, 230-65.

[8] Waldrop MM. The Dream Machine: JCR Licklider and the Revolution that Made Computing Personal. Viking Penguin; 2001.

[9] Engelbrecht A. Computational Intelligence. Chichester: Wiley; 2007.

[10] Russell S, Norvig P. Artificial Intelligence. Upper saddle river: Prentice Hall; 1995.

[11] Turing AM. Computing machinery and intelligence. 1950; Mind, LIX, 433-460.

[12] Bickle J. Multiple realizability. In: E. Zalta (Ed.), Stanford Encyclopaedia of Philosophy. 2020 Summer.

[13] Hollnagel E, Woods DD. Joint cognitive systems: foundations of cognitive systems engineering. Boca Raton, FL: CRC Press; 2005.

[14] Roth EM, Woods DD, Pople HE. Cognitive simulation as a tool for cognitive task analysis. Ergonomics. 1992; 35(10): 1163-1198.

[15] Roth EM, Woods DD. Cognitive Task Analysis: An Approach to Knowledge Acquisition for Intelligent System Design. Studies in Computer Science and Artificial Intelligence. 1989; 5: 233-264.

[16] Newell A, Simon HA. Human Problem Solving. Engelwood Cliffs, NJ: Prentice-Hall; 1972.

[17] Rescorla M. The Computational Theory of Mind. In: E. Zalta (Ed.), Stanford Encyclopaedia of Philosophy. 2020 Fall.

[18] Sun R. Potential of full human-machine symbiosis through truly intelligent cognitive systems. AI \& Society. (2017) 2020 Mar; 35(1): 17-28.

[19] Lieto A. Cognitive Design for Artificial Minds. London and New York: Routledge; 2021.

[20] Saariluoma P. Chess Players' Thinking. London: Routledge; 1995.

[21] Saariluoma P. Foundational Analysis: Presuppositions in Experimental Psychology. London: Routledge; 1997.

[22] Anderson JR. Rules of the Mind. Hillsdale, NJ: Erlbaum; 1993.

[23] Polk T, Seiffert C. Cognitive Modelling. Cambridge, MA: MIT press; 2002.

[24] Broadbent D. Perception and Communication. London: Pergamon Press; 1958.

[25] Miller GA. The magical number seven, plus or minus two: Some limits on our capacity for processing information. Psychological Review. 1956; 63: 81-97.

[26] Shannon CE. A mathematical theory of communication. The Bell System Technical Journal. 1948; 27(3): $379-423$

[27] Aspray WF. The scientific conceptualization of information: A survey. Annals of the History of Computing. 1985; 7(2): 117-140.

[28] Anderson JR. Language, Memory and Thought. Hillsdale, NJ: Erlbaum; 1976.

[29] Johnson-Laird P. Mental Models: Towards a Cognitive Science of Language, Inference, and Consciousness. Cambridge, MA: Harvard University Press; 1983.

[30] Johnson-Laird P. How We Reason. Oxford: Oxford University Press; 2008.

[31] Collins A, Quillian M. Retrieval time from semantic memory. Journal of Verbal Learning and Verbal Behaviour. 1969; 8: 240-248.

[32] Ericsson KA, Simon HA. Protocol Analysis. Cambridge, MA: MIT Press; 1984.

[33] Patton M. Qualitative Evaluation and Research Methods. London: Sage; 1990.

[34] Saariluoma P. Chess and content-oriented psychology of thinking. Psicologica. 2001; 22: 143-164.

[35] Saariluoma P, Nevala K, Karvinen M. Content-based analysis of modes in design engineering. In Design Computing and Cognition. 2006; 325-344.

[36] Vincent JF, Bogatyreva OA, Bogatyrev NR, Bowyer A, Pahl AK. Biomimetics: its practice and theory. Journal of the Royal Society Interface. 2006; 3(9): 471-482.

[37] Saariluoma P, Kujala T, Karvonen A, Ahonen M. Cognitive mimetics Main ideas. Proceedings on the International Conference on Artificial Intelligence (ICAI) 2018:202-206.

[38] Floridi L. What is a philosophical question? Metaphilosophy. 2013; 44(3): 195-221.

[39] de Groot A. Thought and Choice in Chess. The Hague: Mounton; 1965.

[40] Petzold C. The Annotated Turing. Indianapolis: Wiley; 2008.

[41] Boden M. AI. Oxford: Oxford University Press; 2016.

[42] Barricelli BR, Casiraghi E, Fogli D. A survey on digital twin: Definitions, characteristics, applications, and design implications. IEEE Access. 2019; 7: 167653-167671.

[43] Saariluoma P, Cañas J, Karvonen A. Human digital twins and cognitive mimetic. In: Ahram T, Taiar R, Langlois K, Choplin A, editors. Human Interaction, Emerging Technologies and Future Applications III: Proceedings of the 3rd International Conference on Human Interaction and Emerging Technologies: Future Applications (IHIET 2020), 2020 August 27-29; Paris, France. Advances in Intelligent Systems and Computing vol 1253. Springer, Cham; 2021.

[44] Kaivo-oja J, Knudsen MS, Lauraeus T, Kuusi O. Future knowledge management challenges: Digital twins approach and synergy measurements. Management. 2020; 8(2): 99-109. 
[45] Hafez W. Human digital twin: Enabling human-multi smart machines collaboration. In: Bi Y, Bhatia R, Kapoor S, editors. Intelligent Systems and Applications. IntelliSys 2019. Advances in Intelligent Systems and Computing, vol 1038. Springer, Cham; 2019 september.

[46] Floridi L. The Logic of Design as a Conceptual Logic of Information. Minds \& Machines. 2017; 27: 495-519.

[47] Wiener N. Cybernetics or Control and Communication in the Animal and the Machine. Cambridge, MA: MIT Press; 1948.

[48] Särkkä T, Gutiérrez-Poch M, Kuhlberg M. Technological Transformation in the Global Pulp and Paper Industry 1800-2018: Comparative Perspectives. Springer, Cham; 2018.

[49] Bennett S. Nicolas Minorsky and the automatic steering of ships. IEEE Control Systems Magazine. 1984; 4(4): 10-15.

[50] Minorsky N. Directional stability of automatically steered bodies. Journal of the American Society of Naval Engineers. 1922; 34: 280-309.

[51] Selz O. Ueber die Gesetze des geordneten Denkverlaufs [On the laws of organized thinking]. Stuttgart: Spemann; 1913.

[52] Lindsay P, Norman, D. Human Information Processing. New York: Academic Press; 1977. 